\title{
Overview of ethical issues for conducting neuroprotective clinical trials in patients with spinal cord injury
}

This article was published in the following Dove Press journal:

Journal of Neurorestoratology

6 May 2015

Number of times this article has been viewed

\author{
Hooshang Saberi ${ }^{1,2}$ \\ Nazi Derakhshanrad' \\ Mahsa Ghajarzadeh' \\ 'Brain and Spinal Cord Injury \\ Research Center, Imam Khomeini \\ Hospital, ${ }^{2}$ Department of \\ Neurosurgery, Imam Khomeini \\ Hospital, Tehran University of \\ Medical Sciences, Tehran, Iran
}

Correspondence: Hooshang Saberi Brain and Spinal Cord Injury Research Center (BASIR), Imam Khomeini Hospital Complex, Keshavarz Boulevard, PO Box I4I85-6I, Tehran, Iran Tel $+98216658 I 560$

Fax +982I 66938885

Email hgsaberi@yahoo.com

\begin{abstract}
Animals have been used in medical and biological researches worldwide for long time. Almost all of these studies are published with the hope of clinical application. However, there are meticulous criteria considering results of animal studies in clinical trials of human beings. In recent years, the number of experimental research in animal models of spinal cord injury has been growing. However, there is no warranty for translation of experimental studies into clinical practice. Certain protocols should be considered in all phases of conducting clinical trials such as study design, data gathering, and analysis. In this article, we comprehensively review different aspects of ethical issues in translating results of animal studies into clinical application, especially in patients with spinal cord injury.
\end{abstract}

Keywords: neuroprotective treatment, spinal cord injury, animal studies, translation

\section{Introduction}

\section{Problems with translational aspect of spinal cord injury in experimental models}

SCI (spinal cord injury) is a rare condition that affects all aspects of patients' life.

As it is not as common as disorders such as stroke or Alzheimer's disease, there is lower tendency for development and application of new treatments. On the other hand, single therapy is useless in SCI cases and multiple remedies and approaches should be applied simultaneously for these patients. So, detection of the effects of specific therapy is not easily possible. ${ }^{1}$

Animal models are used in SCI research. ${ }^{2}$ However, awareness of the limitations of animal research and its contribution to make reliable predictions about human health is growing. There are several points considering translation of animal studies into clinical trials of human beings. ${ }^{3-5}$ At the same time, ethical issues should be regarded precisely in animal studies, ie, using small instead of large animals if possible at the beginning of the study, using minimum number of animals, and imposing least amount of pain.

In translating results of animal research into human trials, the following points should be considered:

1. Animal studies do not reliably predict human outcomes for the same trial.

2. Relying on only animal experiments can impede and delay discovery, and preclinical phases are required.

3. Animal studies are flawed by design (due to disease modeling).

In recent years, the number of experimental research in animal models of SCI is growing. Those with certain criteria are suitable for translation of experimental studies 
into clinical practice. Certain protocols should be considered in all phases of conducting trials such as study design, data gathering, and analysis. ${ }^{6}$

\section{Ethical issues in experimental research}

The model of neurological injury is of special concern because of possible suffering (especially when considering larger animals with neurological injury). Therefore, rodents are the most preferable model for SCI. At the beginning, the sample size of such studies should not exceed the necessary calculated number (the exact number depends on study design and statistical parameters), ${ }^{7}$ for example, in a previous cat study, only three male cats were enrolled. ${ }^{8,9}$ The painful steps should be performed under sufficient analgesia and anesthesia, and/or the paralyzed animals should be sacrificed when the study is completed (to make sure about prevention of secondary suffering). The animals should not be stressed, and they should not participate in multiple studies.

\section{Limitations of using animals}

Animal studies with particular characters may predict human outcomes. ${ }^{10}$ Although the number of experimental research in animal models of SCI is growing exponentially, prompt translation of experimental studies into clinical practice is not reasonable. Establishment of protocol in large mammals and rodents for evaluating mechanism of action of drugs is essential for translating animal research into human trials. $^{11}$

\section{Ethical issues in clinical research}

One controversial issue in the field of SCI research is whether to give hope to patients or not before enrollment into the study. A number of issues should be explained clearly such as safety issues and complications such as infections, neuropathic pain, spasticity, new cellular growth, and neurological deterioration. ${ }^{12}$ It may be recommended that one should avoid giving false hope to patients and at the same time we should not make patients disappointed.

\section{General items that should be considered}

Minimal invasion is a considerable necessity in translating animal research. If a modality may be accomplished peripherally, it is better not to go for intra-axial injections. For local treatment, based on the size of the transported tissue, minimal incision size should be considered. Therefore, surgeries for delivering the therapeutic cells should be minimally invasive.

\section{Methodological concerns}

The best evidences for establishment of new interventions are double-blind randomized clinical trials. ${ }^{13}$ According to the type and duration of therapy, design of clinical trial may vary. ${ }^{12}$ For instance, neuroprotective therapies would start at early stages of injury (within hours to days of injury such as early spinal cord decompression after SCI), or if secondary damage is targeted, the therapy could be started few days after injury (depending on the particular item of interest). If therapies promote repair or plasticity, they could be started in acute, sub-acute, or chronic stages. ${ }^{12}$ Every trial (such as rehabilitation and education programs and/or cell therapies), to be conducted in any stage, should be supported by previous animal studies. As the chronic phase of the disease is the most static phase of the injury (less neurological improvement), trials targeting this phase will need less number of cases (like Mackay-Sim study for olfactory cells for SCI).,14

\section{Assessment objectivity}

If the study needs paraclinical evaluation such as electrophysiological tests, digital gait analysis, and/or imaging modalities, they should be reported as additional data to the clinical findings like results of digital gait analysis.

Randomization and blinding are two major critical issues in Phase II and III studies. (So, this issue and its ethical concerns like sham surgeries need special concerns.)

To decrease study biases and increase reproducibility of the research results, outcomes of the study should be evaluated by independent observers, and this item should be mentioned in the article.

\section{Cellular procurement standards}

These protocols are among the most important concerns, and they are defined locally depending on the country, according to good manufacturing practice protocols as reported by Food and Drug Administration or Foundation for the Accreditation of Cell Therapy protocols or local governmental declaration. All standards should be regarded in delivering biological materials to the delivery or operating rooms. To reduce the rate of viral and/or autoimmune complications, autologous cells are the best sources. Fetal cells need a great concern according to local laws. The materials used for cellular procurement should also be of acceptable clinical grade, and animal origin materials should be avoided. All these rules need local approval by governmental regulations.

\section{Informed consent}

Informed consent is among the keystones of cellular therapy studies. The treatment and possible expected outcomes 
should be clearly explained to the patient. If any doubts exist, patients should have enough time to discuss the topic with their families and relatives. As much as possible, children or pregnant women, or mentally retarded subjects, should not be included in the study. The informed consents for SCI trials should contain information regarding risk of neuropathic pain, risk of spasticity, risk of autonomic dysreflexia, risk of loss of function, uncertainty of adverse effects, and risk of infection. ${ }^{15}$ These studies should not be performed on prisoners, soldiers, and nursery children. Sham surgery controls, enrollment in trials of uncertain benefit with potential risk, and abortion for obtaining the tissue or cells should also be avoided.

Surgical and rehabilitation standardization, post-trial treatment for control subjects, and compassionate use protocols for the patients should be considered. For example, the study should provide rehabilitation standards for the subjects with SCI in the trial, and if proved, effectiveness has been reported for controls who receive treatment.

Safety issues such as infectious complications and increments in neuropathic pain and/or spasticity and honest report of complications are very important concerns, especially in Phase I studies. These factors need to be reported as basal pretreatment, just after treatment, and follow-up (commonly in the range of 2 years) ${ }^{14,16}$ phases of the study. All positive and negative results should be published clearly.

One important point in designing trials on patients with SCI is injury level in included cases. In selecting cases with specific level of injury, mechanism of drug or cell action, route of administration, and distance of affected spinal segments should be considered. For instance, patients with cervical, lumbar, and lower thoracic spinal cord injuries will benefit more than patients with mid or higher thoracic cord injuries (distant from cervical and lumbar enlargement) from cell graft into the lesion site, which improves axon growth or remyelination in near segments. ${ }^{12}$ At the same time, on the other hand, manipulation of cervical and lumbar spinal cord regions will result in the greatest hazard for undesirable effects due to surgical trauma (not suitable cases for safety studies and/or invasive methods). If the therapy could affect many spinal segments (like systemic drug administration), patients with thoracic injury are better candidates. For instance, administration of growth factors when it is applied in thoracic cases could affect lumbar spine segments and show better results. ${ }^{12}$

It is a better practice to perform early safety studies, in thoracic cases with American Spinal Injury Association impairment scale $\mathrm{A}$, to prevent any disastrous event. ${ }^{14}$
Meanwhile, long-term follow-up, with proper questionnaires and standard measuring scales, for example, American Spinal Injury Association Scale for neurological status, Modified Ashworth Scale for spasticity, Nominal Rating Scale for pain and spinal cord independence measure, and International Association of Neurorestoratology Spinal Cord Injury Functional Rating Scale for report of functional outcomes are essential.

Cost of the study afforded by research grants should be regarded in conducting human trials.

Patients with partial spinal cord injuries have more to lose from surgery than those with complete injuries. So, they are not good candidates for safety phase studies.

Finally, patients, biotechnology companies, and universities with interests in any new area of medical innovation put pressure on surgeons to perform the surgery or treatment as soon as it looks promising. However, clinicians should keep independent for clinical judgment to be done unbiased and their prescriptions and indication should be based on solid scientific evidence.

\section{Conclusion}

The hope with stem cells and neuroprotective treatments for persons with SCI is promising. Adherence to ethical as well as methodological protocols would definitely promote the tempo of scientific field.

\section{Disclosure}

The authors report no conflicts of interest in this work.

\section{References}

1. Karimi-Abdolrezaee S, Eftekharpour E, Wang J, Morshead CM, Fehlings MG. Delayed transplantation of adult neural precursor cells promotes remyelination and functional neurological recovery after spinal cord injury. J Neurosc. 2006;26(13):3377-3389.

2. Matthews RA. Medical progress depends on animal models-doesn't it? $J$ R Soc Med. 2008;101(2):95-98.

3. Hackam DG, Redelmeier DA. Translation of research evidence from animals to humans. JAMA. 2006;296(14):1727-1732.

4. Perel P, Roberts I, Sena E, et al. Comparison of treatment effects between animal experiments and clinical trials: systematic review. $B M J$ 2007;334(7586):197

5. Hackam DG. Translating animal research into clinical benefit. BMJ. 2007;334(7586):163-164.

6. Guest J, Harrop JS, Aarabi B, et al. Optimization of the decision-making process for the selection of therapeutics to undergo clinical testing for spinal cord injury in the North American Clinical Trials Network J Neurosurg Spine. 2012;17(1 Suppl):94-101.

7. Fawcett JW, Curt A, Steeves JD, et al. Guidelines for the conduct of clinical trials for spinal cord injury as developed by the ICCP panel: spontaneous recovery after spinal cord injury and statistical power needed for therapeutic clinical trials. Spinal Cord. 2006;45(3): 190-205.

8. Schaller B, Graf R, Wienhard K, Heiss W. A new animal model of cerebral venous infarction: ligation of the posterior part of the superior sagittal sinus in the cat. Swiss Med Wkly. 2003;133(29/30):412-418. 
9. Schaller B, Graf R, Sanada Y, et al. Hemodynamic changes after occlusion of the posterior superior sagittal sinus: an experimental PET study in cats. Am J Neuroradiol. 2003;24(9):1876-1880.

10. van der Worp HB, Howells DW, Sena ES, et al. Can animal models of disease reliably inform human studies? PLoS Med. 2010;7(3): e1000245.

11. Rosenfeld JV, Bandopadhayay P, Goldschlager T, Brown DJ. The ethics of the treatment of spinal cord injury: stem cell transplants, motor neuroprosthetics, and social equity. Top Spinal Cord Inj Rehabil. 2008;14(1):76-88.

12. Tuszynski MH, Steeves JD, Fawcett JW, et al; International Campaign for Cures of Spinal Cord Injury Paralysis. Guidelines for the conduct of clinical trials for spinal cord injury as developed by the ICCP panel: clinical trial inclusion/exclusion criteria and ethics. Spinal Cord. 2007;45(3):222-231.
13. Fehlings MG, Vawda R. Cellular treatments for spinal cord injury: the time is right for clinical trials. Neurotherapeutics. 2011;8(4): 704-720.

14. Mackay-Sim A, Féron F, Cochrane J, et al. Autologous olfactory ensheathing cell transplantation in human paraplegia: a 3-year clinical trial. Brain. 2008;131(9):2376-2386.

15. Illes J, Reimer JC, Kwon BK. Stem cell clinical trials for spinal cord injury: readiness, reluctance, redefinition. Stem Cell Rev. 2011;7(4): 997-1005.

16. Saberi H, Firouzi M, Habibi Z, et al. Safety of intramedullary Schwann cell transplantation for postrehabilitation spinal cord injuries: 2-year follow-up of 33 cases: clinical article. J Neurosurg Spine. 2011;15(5): $515-525$.

\section{Publish your work in this journal}

The Journal of Neurorestoratology is an international, peer-reviewed, open access online journal publishing original research and review articles on the subject of Neurorestoratology. To provide complete coverage of this revolutionary field the Journal of Neurorestoratology will report on relevant experimental research, technological advances, and

\section{Dovepress}

clinical achievements. The manuscript management system is completely online and includes a very quick and fair peer-review system, which is all easy to use. Visit http://www.dovepress.com/testimonials.php to read real quotes from published authors. 